\title{
Dehn surgeries on knots in product manifolds
}

\author{
Yi NI \\ Department of Mathematics, Caltech, MC 253-37 \\ 1200 E California Blvd, Pasadena, CA 91125 \\ Email: yni@caltech.edu
}

\begin{abstract}
We show that if a surgery on a knot in a product sutured manifold yields the same product sutured manifold, then this knot is a $0-$ or 1crossing knot. The proof uses techniques from sutured manifold theory.
\end{abstract}

Dedicated to the memory of Professor Andrew Lange

\section{Introduction}

An interesting problem on Dehn surgery is: when does a surgery on a knot yield a manifold homeomorphic to the original ambient manifold? The most famous result in this direction is the Knot Complement Theorem proved by Gordon and Luecke [8]: when the ambient manifold is $S^{3}$, only the unknot admits surgeries which yield $S^{3}$.

In this paper, we are going to study this problem for knots in surfaces times an interval. Our main result is as follows.

Theorem 1.1. Suppose $F$ is a compact surface, $K \subset F \times I$ is a knot. Suppose $\alpha$ is a nontrivial slope on $K$, and $N(\alpha)$ is the manifold obtained from $F \times I$ via the $\alpha$-surgery on $K$. If the pair $(N(\alpha),(\partial F) \times I)$ is homeomorphic to the pair $(F \times I,(\partial F) \times I)$, then one can isotope $K$ such that its image on $F$ under the natural projection

$$
p: F \times I \rightarrow F
$$

has either no crossing or exactly one crossing.

The slope $\alpha$ can be determined as follows. Let $\lambda_{b}$ be the "blackboard" frame of $K$ associated with the previous projection. Namely, $\lambda_{b}$ is the frame specified by the surface $F$. When the projection has no crossing, $\alpha=\frac{1}{n}$ for some integer $n$ with respect to $\lambda_{b}$; when the minimal projection has exactly one crossing, $\alpha=\lambda_{b}$.

It is easy to see the surgeries in the statement of Theorem 1.1 do not change the homeomorphism type of the pair $(F \times I,(\partial F) \times I)$. In fact, when $K$ is a

0 -crossing knot, it is clear that the $\frac{1}{n}$-surgery preserves the homeomorphism 


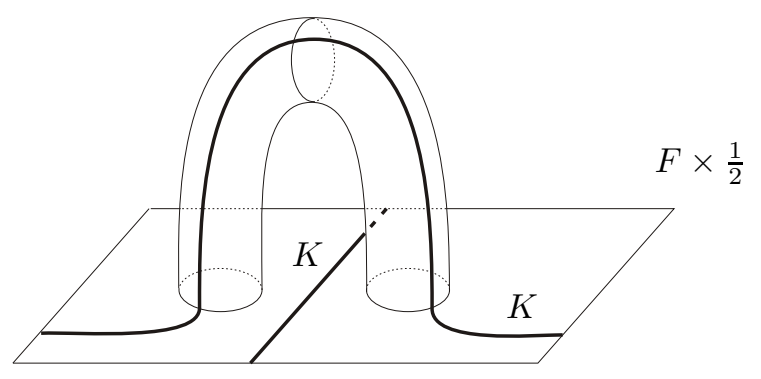

Figure 1: A local picture of the crossing

type of the pair. When $K$ is a 1 -crossing knot, we can add a one-handle to $F \times \frac{1}{2}$ near the crossing to get a Heegaard surface $F^{\prime}$ for $F \times I$. $K$ can be embedded into $F^{\prime}$ as in Figure 1. $F^{\prime}$ splits $F \times I$ into two parts $U_{0}, U_{1}$, where $U_{0}$ is $F \times\left[0, \frac{1}{2}\right]$ with a one-handle added to $F \times \frac{1}{2}$, and $U_{1}$ is $F \times\left[\frac{1}{2}, 1\right]$ with a one-handle added to $-F \times \frac{1}{2}$. The embedding of $K$ can be chosen such that $K$ goes through each of the two one-handles exactly once. Now the blackboard frame $\lambda_{b}$ is the frame specified by $F^{\prime}$, and the $\lambda_{b}$-surgery on $F^{\prime}$ cancels each one-handle with a two-handle. Hence the new pair is still homeomorphic to $(F \times I,(\partial F) \times I)$.

Definition 1.2. Notations are as in the previous theorem. Fix a product structure on $(\partial F) \times I$. Up to an isotopy relative to $(\partial F) \times I$, this product structure uniquely extends to a product structure $\mathcal{P}$ on $F \times I$ and a product structure $\mathcal{P}_{\alpha}$ on $N(\alpha)$. (This fact can be proved using Alexander's trick.) Identify $F$ with $F \times 1$. Let $i, i_{\alpha}: F \times 0 \rightarrow F \times 1$ be the natural identity maps with respect to $\mathcal{P}$ and $\mathcal{P}_{\alpha}$, respectively. We call

$$
\varphi_{\alpha}=i \circ i_{\alpha}^{-1}: F \rightarrow F
$$

the map induced by the $\alpha$-surgery. This map $\varphi_{\alpha}$ fixes $\partial F$ pointwise, and is unique up to an isotopy relative to $\partial F$. Hence $\varphi_{\alpha}$ can be viewed as an element in the mapping class group $\mathcal{M C G}(F, \partial F)$.

The definition of the map $\varphi_{\alpha}$ is justified by the following lemma.

Lemma 1.3. Let $Y(\alpha)$ be the manifold obtained from $F \times S^{1}$ by $\alpha$-surgery on $K$. Then $Y(\alpha)$ can be obtained from $F \times I$ by identifying $(x, 0)$ with $\left(\varphi_{\alpha}(x), 1\right)$ for any $x \in G$.

Proof. The manifold $F \times S^{1}$ is obtained from $F \times I$ by identifying $y$ with $i(y)$ for each $y \in F \times 0$. Let $y=(x, 0)$ with respect to the product structure $\mathcal{P}_{\alpha}$ on $N(\alpha)$, then $i_{\alpha}(y)=(x, 1)$ with respect to $\mathcal{P}_{\alpha}$. We then have

$$
i(y)=\varphi_{\alpha}(x, 1)=\left(\varphi_{\alpha}(x), 1\right),
$$

since we identify $F$ with $F \times 1$ in the above definition. Hence $(x, 0)$ is identified with $\left(\varphi_{\alpha}(x), 1\right)$ in $Y(\alpha)$ for each $x \in F$. 


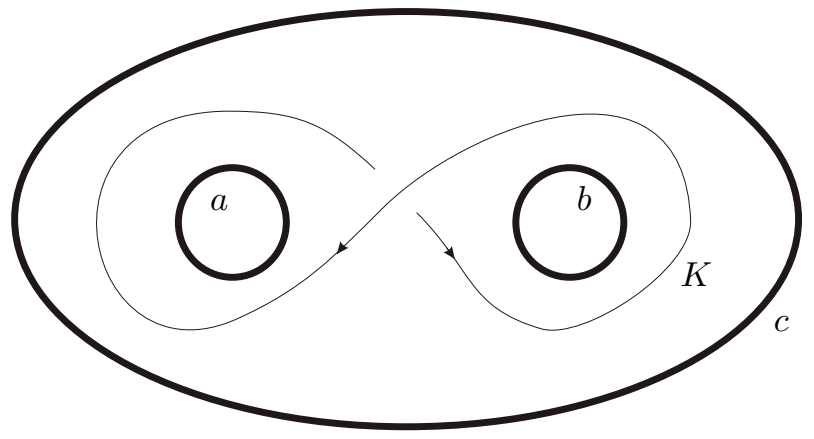

Figure 2: A 1-crossing knot

Proposition 1.4. Notations are as in Theorem 1.1. When the projection of $K$ has no crossing and $\alpha=\frac{1}{n}$,

$$
\varphi_{\alpha}=\tau^{n},
$$

where $\tau$ is the righthand Dehn twist along $K \subset F$. When the minimal projection of $K$ has exactly one crossing, let $a, b, c$ be the simple closed curves obtained by resolving the crossing in two different ways as in Figure 0 and let $\tau_{a}, \tau_{b}, \tau_{c}$ be the righthand Dehn twists along $a, b, c$. Then

$$
\varphi_{\alpha}=\tau_{a}^{2} \tau_{b}^{2} \tau_{c}^{-1}
$$

when the crossing is positive, and

$$
\varphi_{\alpha}=\tau_{a}^{-2} \tau_{b}^{-2} \tau_{c}
$$

when the crossing is negative.

This paper can be compared with Ni [9]. In fact, Theorem 1.4 in [9] can be restated in a form similar to Theorem 1.1 .

Theorem 1.5. Suppose $F$ is a compact surface, $K \subset F \times I$ is a knot and $\alpha$ is a slope on $K$. Let $N(\alpha)$ be the manifold obtained by the $\alpha$-surgery on $K$. If $F \times\{0\}$ is not Thurston norm minimizing in $H_{2}(N(\alpha),(\partial F) \times I)$, then there is an ambient isotopy of $F \times I$ which takes $K$ to a curve in $F \times\left\{\frac{1}{2}\right\}$. Moreover, $\alpha$ is the frame on $K$ specified by $F \times\left\{\frac{1}{2}\right\}$.

The proof of Theorem 1.5 uses Gabai's sutured manifold theory [2, 3, 4, and an argument due to Ghiggini [7. Using a different method, Scharlemann and Thompson [12 get the same conclusion of Theorem 1.5 under the assumption that $F \times\{0\}$ is compressible in $N(\alpha)$.

This paper is organized as follows. In Section 2, we give some preliminaries on sutured manifold theory and foliations, as well as a characterization of onecrossing knot projections. In Section 3, we study some warm-up cases. In Section 4, we use the argument in the proof of Theorem [1.5] to reduce our 
problem to the case where $F$ is a pair of pants. In Section 5 , we study this case by analyzing the map induced by surgery and using a variant of the argument in $\mathrm{Ni}[9$.

Acknowledgements. We are grateful to Danny Calegari for helpful discussions on mapping class groups. The author was partially supported by an AIM Five-Year Fellowship and NSF grant number DMS-0805807.

\section{Preliminaries}

In this section, we are going to review the sutured manifold theory introduced by Gabai in 2. We also state a uniqueness result for the Euler classes of taut foliations of fibred manifolds. In addition, we define "double primitive" knots in $F \times I$ and show that they are exactly the knots with a projection consisting of only one crossing.

\subsection{Sutured manifold decompositions}

Definition 2.1. A sutured manifold $(M, \gamma)$ is a compact oriented 3-manifold $M$ together with a set $\gamma \subset \partial M$ of pairwise disjoint annuli $A(\gamma)$ and tori $T(\gamma)$. The core of each component of $A(\gamma)$ is a suture, and the set of sutures is denoted by $s(\gamma)$.

Every component of $R(\gamma)=\partial M-\operatorname{int}(\gamma)$ is oriented. Define $R_{+}(\gamma)$ (or $\left.R_{-}(\gamma)\right)$ to be the union of those components of $R(\gamma)$ whose normal vectors point out of (or into) $M$. The orientations on $R(\gamma)$ must be coherent with respect to $s(\gamma)$, hence every component of $A(\gamma)$ lies between a component of $R_{+}(\gamma)$ and a component of $R_{-}(\gamma)$.

Definition 2.2. Let $S$ be a compact oriented surface with connected components $S_{1}, \ldots, S_{n}$. We define

$$
x(S)=\sum_{i} \max \left\{0,-\chi\left(S_{i}\right)\right\} .
$$

Let $M$ be a compact oriented 3-manifold, $A$ be a compact codimension-0 submanifold of $\partial M$. Let $h \in H_{2}(M, A)$. The Thurston norm $x(h)$ of $h$ is defined to be the minimal value of $x(S)$, where $S$ runs over all the properly embedded surfaces in $M$ with $\partial S \subset A$ and $[S]=h$.

Definition 2.3. Let $(M, \gamma)$ be a sutured manifold, and $S$ a properly embedded surface in M, such that no component of $\partial S$ bounds a disk in $R(\gamma)$ and no component of $S$ is a disk with boundary in $R(\gamma)$. Suppose that for every component $\lambda$ of $S \cap \gamma$, one of 1)-3) holds:

1) $\lambda$ is a properly embedded non-separating arc in $\gamma$.

2) $\lambda$ is a simple closed curve in an annular component $A$ of $\gamma$ in the same homology class as $A \cap s(\gamma)$. 
3) $\lambda$ is a homotopically nontrivial curve in a toral component $T$ of $\gamma$, and if $\delta$ is another component of $T \cap S$, then $\lambda$ and $\delta$ represent the same homology class in $H_{1}(T)$.

Then $S$ is called a decomposing surface, and $S$ defines a sutured manifold decomposition

$$
(M, \gamma) \stackrel{S}{\rightsquigarrow}\left(M^{\prime}, \gamma^{\prime}\right),
$$

where $M^{\prime}=M-\operatorname{int}(\operatorname{Nd}(S))$ and

$$
\begin{aligned}
\gamma^{\prime} & =\left(\gamma \cap M^{\prime}\right) \cup \operatorname{Nd}\left(S_{+}^{\prime} \cap R_{-}(\gamma)\right) \cup \operatorname{Nd}\left(S_{-}^{\prime} \cap R_{+}(\gamma)\right), \\
R_{+}\left(\gamma^{\prime}\right) & =\left(\left(R_{+}(\gamma) \cap M^{\prime}\right) \cup S_{+}^{\prime}\right)-\operatorname{int}\left(\gamma^{\prime}\right), \\
R_{-}\left(\gamma^{\prime}\right) & =\left(\left(R_{-}(\gamma) \cap M^{\prime}\right) \cup S_{-}^{\prime}\right)-\operatorname{int}\left(\gamma^{\prime}\right),
\end{aligned}
$$

where $S_{+}^{\prime}\left(S_{-}^{\prime}\right)$ is that component of $\partial \mathrm{Nd}(S) \cap M^{\prime}$ whose normal vector points out of (into) $M^{\prime}$.

Definition 2.4. A sutured manifold $(M, \gamma)$ is taut, if $M$ is irreducible and $R(\gamma)$ is Thurston norm minimizing in $H_{2}(M, \gamma)$.

Suppose $S$ is a decomposing surface in $(M, \gamma), S$ decomposes $(M, \gamma)$ to $\left(M^{\prime}, \gamma^{\prime}\right) . S$ is taut if $\left(M^{\prime}, \gamma^{\prime}\right)$ is taut.

Definition 2.5. Suppose

$$
(M, \gamma) \stackrel{S}{\rightsquigarrow}\left(M^{\prime}, \gamma^{\prime}\right)
$$

is a taut decomposition, by 2 we can extend this decomposition to a sutured manifold hierarchy of $(M, \gamma)$, from which we can construct a taut foliation $\mathscr{F}$ of $M$, such that $R(\gamma)$ consists of compact leaves of $\mathscr{F}$. We then call $\mathscr{F}$ a foliation induced by $S$. Moreover, when $R_{+}(\gamma)$ is homeomorphic to $R_{-}(\gamma)$, from $M$ we can obtain a manifold $Y$ with boundary consisting of tori by gluing $R_{+}(\gamma)$ to $R_{-}(\gamma)$ via a homeomorphism. $\mathscr{F}$ then becomes a taut foliation $\mathscr{F}_{1}$ of $Y$. We also say that $\mathscr{F}_{1}$ is a foliation induced by $S$.

Definition 2.6. A decomposing surface is called a product disk, if it is a disk which intersects $s(\gamma)$ in exactly two points. A decomposing surface is called a product annulus, if it is an annulus with one boundary component in $R_{+}(\gamma)$, and the other boundary component in $R_{-}(\gamma)$.

We recall the main result in Gabai [3, which has been intensively used in $\mathrm{Ni}$ 9. Note that the result is not stated in its original form, but it is contained in the argument in [3]. See also [9, Theorem 2.8] for a sketch of the proof.

Definition 2.7. An I-cobordism between closed connected surfaces $T_{0}$ and $T_{1}$ is a compact 3-manifold $V$ such that $\partial V=T_{0} \cup T_{1}$ and for $i=0,1$ the induced maps $j_{i}: H_{1}\left(T_{i}\right) \rightarrow H_{1}(V)$ are injective.

Definition 2.8. Suppose $M$ is a 3 -manifold, $T$ is a toral component of $\partial M$. If all tori in $M$ which are I-cobordant to $T$ in $M$ must be parallel to $T$, then we say $M$ is $T$-atoroidal. 
Theorem 2.9 (Gabai). Let $(M, \gamma)$ be a taut sutured 3-manifold. $T$ is a toral component of $\gamma, S$ is a decomposing surface such that $S \cap T=\emptyset$, and the decomposition

$$
(M, \gamma) \stackrel{S}{\rightsquigarrow}\left(M_{1}, \gamma_{1}\right)
$$

is taut. Suppose $M$ is $T$-atoroidal, then for any slope $\alpha$ on $T$ except at most one slope, the decomposition after Dehn filling

$$
(M(\alpha), \gamma \backslash T) \stackrel{S}{\rightsquigarrow}\left(M_{1}(\alpha), \gamma_{1} \backslash T\right)
$$

is taut.

A special case of the above theorem is the case $\gamma=\partial M$, which is the original form in 3 .

\section{$2.2 \quad$ Euler classes of foliations}

We will need the Euler classes of foliations.

Definition 2.10. Suppose $Y$ is a compact 3-manifold with $\partial Y$ consisting of tori. $\mathscr{P}$ is an oriented plane field transverse to $\partial Y$. Let $T(\partial Y)$ be the tangent plane field of $\partial Y$. The line field $\mathscr{P} \cap T(\partial Y)$ has a natural orientation induced by the orientations of $\mathscr{P}$ and $T(\partial Y)$, thus it has a nowhere vanishing section $\left.v \subset \mathscr{P}\right|_{\partial Y}$. Then one can define the relative Euler class

$$
e(\mathscr{P}) \in H^{2}(Y, \partial Y)
$$

of $\mathscr{P}$ to be the obstruction to extending $v$ to a nowhere vanishing section of $\mathscr{P}$. When $\mathscr{F}$ is a foliation of $Y$ that is transverse to $\partial Y$, let $T \mathscr{F}$ be the tangent plane field of $\mathscr{F}$ and let $e(\mathscr{F})=e(T \mathscr{F})$.

Definition 2.11. Suppose $C$ is a properly embedded curve in a compact surface $F$. We say $C$ is efficient in $F$ if

$$
|C \cap \delta|=|[C] \cdot[\delta]|, \quad \text { for each boundary component } \delta \text { of } F .
$$

Suppose $S$ is a properly embedding surface in compact 3-manifold $Y$ with boundary consisting of tori. We say $S$ is efficient in $Y$ if $S \cap T$ consists of coherently oriented parallel essential curves for each boundary component $T$ of $Y$.

Proposition 2.12. Suppose $Y$ is a compact 3-manifold that fibres over $S^{1}$. Let $G$ be a fibre of the fibration $\mathscr{E}$. Suppose $\mathscr{F}$ is a taut foliation of $Y$ which is transverse to $\partial Y$ such that $G$ is a leaf of $\mathscr{F}$. Then

$$
e(\mathscr{F})=e(\mathscr{E}) \in H^{2}(Y, \partial Y) / \text { Tors. }
$$


Proof. This result follows easily from the fact that the Floer homology of a fibred manifold is "monic". Using this approach, one can even prove that the two Euler classes are equal in $H^{2}(Y, \partial Y)$. Here we will present a more geometric proof.

In order to prove the desired result, we only need to show that

$$
\langle e(\mathscr{F}), h\rangle=\langle e(\mathscr{E}), h\rangle
$$

for any $h \in H_{2}(Y, \partial Y)$. When $h=[G]$, we have

$$
\langle e(\mathscr{F}),[G]\rangle=\langle e(\mathscr{E}),[G]\rangle=\chi(G) .
$$

In general, suppose $\bar{U} \subset Y$ is a proper surface representing $h$ such that $\bar{U} \pitchfork G$. We can choose the representative $\bar{U}$ such that $\bar{U}$ is efficient in $Y$. Then $\bar{U} \cap G$ can also be made efficient in $G$. Cutting $Y$ open along $G$, we get $G \times I$. Let $U \subset G \times I$ be the proper surface obtained by cutting $\bar{U}$ open along $C=\bar{U} \cap G$. Let $C_{0}, C_{1} \subset G$ be proper oriented curves such that

$$
-C_{0} \times 0=(\partial U) \cap(G \times 0), \quad C_{1} \times 1=(\partial U) \cap(G \times 1) .
$$

Since $C_{0}$ and $C_{1}$ are homologous efficient curves in $G$ relative to $\partial G$, as in the proof of [3, Lemma 0.6], we can find compact subsurfaces $V_{1}, V_{2}, \ldots, V_{n}$ and efficient curves

$$
C_{0}=\gamma_{0}, \gamma_{1}, \ldots, \gamma_{n-1}, \gamma_{n}=C_{1}
$$

in $G$, such that

$$
\overline{\partial V_{i} \backslash(\partial G)}=\gamma_{i} \cup\left(-\gamma_{i-1}\right) .
$$

Let $W_{i}=\overline{G \backslash V_{i}}$. Perturbing the surface

$$
\bigcup_{i=1}^{n}\left(\left(-\gamma_{i-1} \times\left[\frac{i-1}{n}, \frac{i}{n}\right]\right) \cup\left(V_{i} \times \frac{i}{n}\right)\right)
$$

slightly, we get a proper surface $V \subset G \times I$, such that

$$
(\partial V) \cap(G \times 0)=-C_{0} \times 0, \quad(\partial V) \cap(G \times 1)=C_{1} \times 1 .
$$

Similarly, perturbing the surface

$$
\bigcup_{i=1}^{n}\left(\left(\gamma_{i-1} \times\left[\frac{i-1}{n}, \frac{i}{n}\right]\right) \cup\left(W_{i} \times \frac{i}{n}\right)\right)
$$

slightly, we have a proper surface $W \subset G \times I$, such that

$$
(\partial W) \cap(G \times 0)=C_{0} \times 0, \quad(\partial W) \cap(G \times 1)=-C_{1} \times 1 .
$$

Let $\bar{V} \subset Y$ be the proper surface obtained from $V$ by identifying $C_{0} \times 0$ and $C_{1} \times 1$ with $C \subset G \subset Y$. Similarly, define $\bar{W} \subset Y$. Note that

$$
[\bar{V}]-[\bar{U}]=[V \cup(-U)] \in H_{2}(Y, \partial Y) .
$$


Perturbing $V \cup(-U)$ slightly, we get a properly immersed surface in $Y$ which is disjoint from the fibre $G$. So $[V \cup(-U)]=m[G]$ for some integer $m$. Using (2), in order to check (11) for $h=[\bar{U}]$, we only need to check it for $h=[\bar{V}]$.

Since $\mathscr{F}$ is taut, by Thurston [13, Corollary 1] we have

$$
\begin{aligned}
\chi(\bar{V}) & \leq\langle e(\mathscr{F}),[\bar{V}]\rangle, \\
\chi(\bar{W}) & \leq\langle e(\mathscr{F}),[\bar{W}]\rangle .
\end{aligned}
$$

Adding the two inequalities together, we get

$$
\chi(\bar{V})+\chi(\bar{W}) \leq\langle e(\mathscr{F}),[\bar{V}]+[\bar{W}]\rangle .
$$

By the constructions (3), (4), the result of doing oriented cut-and-pastes to $\bar{V}$ and $\bar{W}$ is $n$ copies of $G$. So the left hand side of (5) is $n \chi(G)$, while the right hand side is $\langle e(\mathscr{F}), n[G]\rangle=n \chi(G)$. So the equality holds. In particular, we should have

$$
\chi(\bar{V})=\langle e(\mathscr{F}),[\bar{V}]\rangle .
$$

The same argument shows that

$$
\chi(\bar{V})=\langle e(\mathscr{E}),[\bar{V}]\rangle,
$$

so (11) holds for $h=[\bar{V}]$. Since we have checked (11) for all elements $h \in$ $H_{2}(Y, \partial Y), e(\mathscr{F})$ is equal to $e(\mathscr{E})$ up to a torsion element in $H^{2}(Y, \partial Y)$.

\subsection{A characterization of one-crossing knot projections}

In this subsection, we will give a characterization of one-crossing knot projections in terms of double primitive knots. This fact is not used in the current paper, but it is useful to bare it in mind.

Definition 2.13. Let $F^{\prime} \subset F \times I$ be a connected surface of genus $g(F)+1$, and $\partial F^{\prime}=(\partial F) \times \frac{1}{2}$. Suppose $F^{\prime}$ is a Heegaard surface. Namely, $F^{\prime}$ splits $F \times I$ into two parts $U_{0}$ and $U_{1}$, such that $U_{0}$ is homeomorphic to $\left(F \times\left[0, \frac{1}{2}\right]\right) \cup H_{1}$, and $U_{2}$ is homeomorphic to $\left(F \times\left[\frac{1}{2}, 1\right]\right) \cup H_{2}$, where $H_{1}$ is a one-handle with feet on $F \times \frac{1}{2}$ and $H_{2}$ is a one-handle with feet on $-F \times \frac{1}{2}$. A knot $K \subset F \times I$ is a double primitive knot if it is isotopic to a curve on $F^{\prime}$ which goes through each of $H_{1}, H_{2}$ exactly once.

Lemma 2.14. A knot $K \subset F \times I$ is double primitive if and only if it has a projection which has only one crossing.

Proof. If a knot has a one-crossing projection, then it is double primitive as shown in the introduction. Now assume $K$ is double primitive, then $K$ is embedded into a Heegaard surface $F^{\prime}$ as in the above definition.

We claim that $F^{\prime}$ is stabilized. Namely, there is a compressing disk $D_{0} \subset U_{0}$ and a compressing disk $D_{1} \subset U_{1}$ such that $\left|\left(\partial D_{0}\right) \cap\left(\partial D_{1}\right)\right|=1$. When $F$ is closed, this follows from the theorem of Scharlemann and Thompson 11] that the Heegaard splittings of $F \times I$ are standard. When $F$ is not closed, let $R$ be 
the torus with one hole, we can glue a copy of $R$ to each component of $\partial F$, then $F$ becomes a closed surface $G$ and $F^{\prime}$ becomes a Heegaard surface $G^{\prime}$ in $G \times I$. Using Scharlemann and Thompson's theorem, $G^{\prime}$ is stabilized, hence there are compressing disks $D_{0}$ and $D_{1}$ in the two compression bodies separated by $G^{\prime}$, such that $\left|\left(\partial D_{0}\right) \cap\left(\partial D_{1}\right)\right|=1$. Using standard arguments we can isotope $D_{0}$ and $D_{1}$ to be disjoint from the copies of $R \times I$, so $D_{0} \subset U_{0}, D_{1} \subset U_{1}$, thus our conclusion follows.

Since $g\left(F^{\prime}\right)=g(F)+1$, after compressing $F^{\prime}$ along $D_{0}$ we get a surface homeomorphic to $F$ (and hence parallel to $F \times 0$ in $F \times I$ ). So $F^{\prime}$ is obtained from $F \times \frac{1}{2}$ by adding a one-handle, and $D_{1}$ is a disk whose boundary goes through the one-handle exactly once. Now the local picture of $F^{\prime}$ looks exactly like in Figure 1. The knot $K$ goes through the one-handle once and intersects $\partial D_{1}$ once, so there is a crossing near $D_{1}$ and no crossing elsewhere.

\section{Warm-up cases}

In this section, we are going to prove some easy cases of our main theorem. When $F$ is a disk or sphere, our result follows from Gordon and Luecke's Knot Complement Theorem [8]. When $F$ is an annulus, we have the following lemma.

Lemma 3.1. Theorem 1.1 is true when $F$ is an annulus.

Proof. Let $\mathcal{M}$ be the meridian of the solid torus $V=F \times I$, and let $\mathcal{L}$ be the frame of $V$ specified by $\partial F$. By Gabai [5, if $K$ is nontrivial, then $K$ is a $0-$ or 1-bridge braid in $F \times I$.

Capping off one boundary component of $F$ with a disk, we get a disk $D$. Let $\lambda$ be the Seifert frame of $K$ in $D \times I$ and let $\mu$ be the meridian of $K$.

If $K$ is the core of $V$, then the surgery preserves the homeomorphism type of $(F \times I,(\partial F) \times I)$ if and only if the slope is $\mu+n \lambda$ for some integer $n$.

From now on we assume the braid index of $K$ is greater than 1 .

If $K$ is a 0 -bridge braid, then $K$ is isotopic to $p \mathcal{L}+q \mathcal{M}$ on $\partial V$ for some $p, q \in \mathbb{Z}$. Let $\Lambda$ be the frame on $K$ specified by $\partial V$, then $\Lambda=p q \mu+\lambda$. A surgery on $K$ yields a solid torus if and only if the slope $\alpha$ of the surgery satisfies that $\Delta(\alpha, \Lambda)=1$, namely, when the slope $\alpha$ is $\mu+n \Lambda$ for some integer $n$. Now $p \alpha=p \mu+p n \Lambda$ is homologous to $\mathcal{M}+p n(p \mathcal{L}+q \mathcal{M})$ in $V \backslash K$, so the meridian of the new ambient solid torus after surgery is $(1+p q n) \mathcal{M}+p^{2} n \mathcal{L}$. Since the surgery preserves the homeomorphism type of the pair $(F \times I,(\partial F) \times I)$, we must have $\Delta\left((1+p q n) \mathcal{M}+p^{2} n \mathcal{L}, \mathcal{L}\right)=1$, thus $1+p q n= \pm 1$. Since $p>1, n \neq 0$, we have $(p, q, n)=(2,1,-1)$ or $(2,-1,1)$. When $(p, q)=(2,1)$, the slope $\alpha$ on $K$ is

$$
\mu+n(p q \mu+\lambda)=(1+p q n) \mu+n \lambda,
$$

which is 1 with respect to the frame $\lambda$, and the meridian of the new ambient solid torus is $\mathcal{M}+4 \mathcal{L}$; when $(p, q)=(2,-1)$, the slope $\alpha$ on $K$ is -1 , and the meridian of the new ambient solid torus is $\mathcal{M}-4 \mathcal{L}$. We can check $\alpha$ is the blackboard frame. 
If $K$ is a 1 -bridge braid, then $K$ is determined by 3 parameters $\omega, b, t$ by Gabai [6. Here $\omega>0$ is the braid index, $1 \leq b \leq \omega-2, t \equiv r(\bmod \omega)$ for some integer $r$ with $1 \leq r \leq \omega-2$. Since the $\alpha$-surgery yields a solid torus, by [6. Lemma 3.2] the slope of the surgery is $\lambda-(t \omega+d) \mu$, where $d \in\{b, b+1\}$. So $t \omega+d= \pm 1$, which is impossible for any $\omega, b, t$ satisfying the previous restrictions.

Lemma 3.2. In the above lemma, let $\varphi_{\alpha} \in \mathcal{M C G}(F, \partial F)$ be the map induced by the $\alpha$-surgery. If $K$ is the core of $F \times I$ and $\alpha=\frac{1}{n}$, then $\varphi_{\alpha}=\tau^{n}$, where $\tau$ is the right hand Dehn twist in $F$; if $K$ is the $(2, \pm 1)$-cable in $F \times I$, then $\varphi_{\alpha}=\tau^{ \pm 4}$.

Proof. When $K$ is the core of $F \times I$, the conclusion is well-known. When $K$ is the $(2, \pm 1)$-cable, then from the proof of the previous lemma we know that the meridian of the new ambient solid torus is $\mathcal{M} \pm 4 \mathcal{L}$, hence the conclusion follows from the first case.

The following lemma is obvious.

Lemma 3.3. Suppose $(C \times I) \subset(F \times I)$ is a product disk or product annulus, $(C \times I) \cap K=\emptyset$. Let $F_{1}$ be the surface obtained from $F$ by cutting $F$ open along $C$, let $N_{1}$ be the manifold obtained from $N=(F \times I) \backslash \operatorname{int}(\operatorname{Nd}(K))$ by cutting $N$ open along $C \times I$. Then the pair $(N(\alpha),(\partial F) \times I)$ is homeomorphic to $(F \times I,(\partial F) \times I)$ if and only if the pair $\left(N_{1}(\alpha),\left(\partial F_{1}\right) \times I\right)$ is homeomorphic to $\left(F_{1} \times I,\left(\partial F_{1}\right) \times I\right)$.

Lemma 3.4. Theorem 1.1 is true when $F$ is a torus.

Proof. Let $C \subset F$ be a simple closed curve such that $K$ is homologous to a multiple of $C$. Consider the homology class $[C \times I] \in H_{2}(F \times I, \partial(F \times I))$, then $[C \times I] \cdot[K]=0$. It follows that $[C \times I]$ is also a homology class in $H_{2}((F \times I) \backslash K, \partial(F \times I))$.

Let $(S, \partial S) \subset((F \times I) \backslash K, \partial(F \times I))$ be a taut surface representing $[C \times I]$. By Theorem 2.9, $S$ remains taut in at least one of the original $F \times I$ and $N(\alpha) \cong F \times I$. Hence $S$ must be a product annulus. Cutting $F \times I$ open along $S, K$ becomes a knot in (annulus $\times I$ ). Now we can apply Lemma 3.1 and Lemma 3.3 to get our conclusion.

Lemma 3.5. If the conclusion of Theorem 1.1 holds for all knots whose exterior are $\partial(\operatorname{Nd}(K))$-atoroidal, then the conclusion holds for all knots in $F \times I$.

Proof. By the assumption, we only need to consider the case where there is a torus in $N=F \times I \backslash \operatorname{int}(\mathrm{Nd}(K))$ which is I-cobordant but not parallel to $\partial \mathrm{Nd}(K)$. Let $R$ be an "innermost" such torus.

By [9, Lemma 3.1], $R$ bounds a solid torus $U$ in $F \times I$, such that $K \subset U$. Since $R$ is innermost in $N$, if a torus in $(F \times I) \backslash \operatorname{int}(U)$ is I-cobordant to $\partial U=R$, then this torus is parallel to $R$. Let $V$ be the manifold obtained from $U$ by $\alpha-$ surgery on $K$.

By Gabai [5], one of the following cases holds. 
1) $V=D^{2} \times S^{1}$. In this case $K$ is a 0 -bridge or 1 -bridge braid in $U$, and the core $K^{\prime}$ of the surgery is also a 0 -bridge or 1 -bridge braid in $V$. Moreover, $K$ and $K^{\prime}$ have the same braid index $\omega$.

2) $V=\left(D^{2} \times S^{1}\right) \# W$, where $W$ is a closed 3 -manifold and $1<\left|H_{1}(W)\right|<\infty$.

3) $V$ is irreducible and $\partial V$ is incompressible.

Since $V \subset N(\alpha) \cong F \times I$, Cases 2) and 3) can not happen, so the only possible case is 1$)$. Thus the core of $U$ is a knot such that a surgery on the knot yields the pair $(N(\alpha),(\partial F) \times I)$ which is homeomorphic to $(F \times I,(\partial F) \times I)$. Moreover, $N \backslash \operatorname{int}(U)$ is $\partial U$-atoroidal. By our assumption, the core of $U$ is a 0 -crossing or 1 -crossing knot in $F \times I$.

If the core of $U$ is isotopic to $\eta \times\left\{\frac{1}{2}\right\}$ for some simple closed curve $\eta \subset F$, let $G \subset F$ be a tubular neighborhood of $\eta$, then $K$ lies in $G \times I$ after an isotopy. Let $M=(G \times I) \backslash \operatorname{int}(\mathrm{Nd}(K))$. By Lemma 3.3. $(M(\alpha),(\partial G) \times I)$ is homeomorphic to $(G \times I,(\partial G) \times I)$. Applying Lemma 3.1, we find that $K$ is the $(2, \pm 1)-$ cable of the core of $G \times I$, and the slope $\alpha$ is the blackboard frame $\lambda_{b}$.

If the core of $U$ is a 1 -crossing knot, then the blackboard frame $\lambda_{b}^{\prime}$ on $\partial U$ is the meridian of $V$, so $\lambda_{b}^{\prime}$ cobounds a punctured disk with $\omega$ oriented copies of $\alpha$ in $U \backslash \operatorname{int}(\operatorname{Nd}(K))$. Moreover, the meridian $\mu^{\prime}$ on $\partial U$ cobounds a punctured disk with $\omega$ oriented copies of $\mu$ in $U \backslash \operatorname{int}(\operatorname{Nd}(K))$. Since $\left[\lambda_{b}^{\prime}\right] \cdot\left[\mu^{\prime}\right]=1$, considering the intersection of the two punctured disks we conclude that $\omega=1$. Hence $\partial U$ is parallel to $\partial \mathrm{Nd}(K)$, a contradiction.

In light of the above lemma, from now on we assume the exterior of the knot $K$ is $\partial(\mathrm{Nd}(K))$-atoroidal.

\section{Comparing Euler classes of foliations}

Let $E$ be a maximal (up to isotopy) compact essential subsurface of $F$, such that $K$ can be isotoped in $F \times I$ to be disjoint from $E \times I$. Let $G=\overline{F \backslash E}$.

The goal of this section is to prove the following proposition.

Proposition 4.1. The subsurface $G$ is either an annulus or a pair of pants.

Let $T=\partial(\operatorname{Nd}(K)), \gamma=((\partial G) \times I) \cup T$. Let

$$
N=(F \times I) \backslash \operatorname{int}(\mathrm{Nd}(K)), M=(G \times I) \backslash \operatorname{int}(\mathrm{Nd}(K)) .
$$

Then the sutured manifold $(M, \gamma)$ contains no product disks or product annuli. For a proper surface $S \subset M$, let $\partial_{i}(S)=S \cap(G \times i), i=0,1$.

Let $X=\left(G \times S^{1}\right) \backslash \operatorname{int}(\operatorname{Nd}(K))$ be the manifold obtained from $M$ by gluing $G \times 1$ to $G \times 0$ via the identity map of $G$. Suppose $\xi$ is a slope on $K$. Let $N(\xi), M(\xi), X(\xi)$ be the manifolds obtained from $N, M, X$ by $\xi$-filling on $T$, respectively. Let $K(\xi) \subset M(\xi)$ be the core of the new solid torus.

By Lemma 3.3. $X(\xi)$ is a surface bundle over $S^{1}$ with fibre $G$ when $\xi=$ $\infty$ or $\alpha$. We then let $\mathscr{E}(\xi)$ be the fibration of $X(\xi)$. 
Lemma 4.2. $K \subset F \times I$ is as in Theorem 1.1. $N$ is T-atoroidal. Suppose $S \subset M$ is a taut surface such that $S \cap T=\emptyset$ and there exists a curve $C \subset F$ with $\partial_{0} S=-C \times 0, \partial_{1} S=C \times 1$. Let $\bar{S} \subset X$ be the surface obtained from $S$ by gluing $\partial_{0} S$ to $\partial_{1} S$ via the identity map. Let $\mathscr{F}$ be a taut foliation of $X$ induced by $S$. Then

$$
\langle e(\mathscr{F}),[\bar{S}]\rangle=\langle e(\mathscr{E}(\xi)),[\bar{S}]\rangle=\chi(\bar{S})
$$

for some $\xi \in\{\infty, \alpha\}$.

Proof. By Theorem 2.9. $S$ remains taut in $M(\xi)$ for some $\xi \in\{\infty, \alpha\}$. Let $\mathscr{F}^{\prime}$ be a taut foliation of $X(\xi)$ induced by $S$. By Proposition 2.12

$$
e\left(\mathscr{F}^{\prime}\right)=e(\mathscr{E}(\xi)) \in H^{2}(X(\xi), \partial X(\xi) ; \mathbb{Q}) .
$$

Since both $\mathscr{F}$ and $\mathscr{F}^{\prime}$ are induced by $S$, we have

$$
\begin{aligned}
\chi(\bar{S}) & =\langle e(\mathscr{F}),[\bar{S}]\rangle \\
& =\left\langle e\left(\mathscr{F}^{\prime}\right),[\bar{S}]\right\rangle \\
& =\langle e(\mathscr{E}(\xi)),[\bar{S}]\rangle .
\end{aligned}
$$

Proposition 4.3. $K \subset F \times I$ is as in Theorem 1.1. $N$ is T-atoroidal. The inclusion $K \subset G \times I$ induces a map

$$
i_{*}: H_{1}(K ; \mathbb{Q}) \rightarrow H_{1}(G ; \mathbb{Q}) .
$$

Let

$$
\mathcal{V}=\left\{v \in H_{1}(G, \partial G ; \mathbb{Q}) \mid v \cdot i_{*}[K]=0\right\} .
$$

Then the dimension of $\mathcal{V}$ is at most 1.

Let

$$
\rho_{\xi}: H^{2}(X, \partial X ; \mathbb{Q}) \rightarrow H^{2}(X(\xi), \partial X(\xi) ; \mathbb{Q})
$$

be the map induced by the map of pairs

$$
(X(\xi), \partial X(\xi)) \rightarrow(X(\xi),(\partial X(\xi)) \cup K(\xi))
$$

Lemma 4.4. Notations are as in Proposition 4.3. If the dimension of $\mathcal{V}$ is greater than 1 , then there exists a properly embedded surface $H \subset X$ such that

1) $H$ is not a multiple of $[G]$,

2) $H \cap T=\emptyset$,

3) for any two elements $\varepsilon_{\infty} \in \rho_{\infty}^{-1}(e(\mathscr{E}(\infty))), \varepsilon_{\alpha} \in \rho_{\alpha}^{-1}(e(\mathscr{E}(\alpha)))$, we have

$$
\left\langle\varepsilon_{\infty},[H]\right\rangle=\left\langle\varepsilon_{\alpha},[H]\right\rangle
$$


Proof. There is a natural injective map

$$
\sigma: H_{1}(G, \partial G) \rightarrow H_{2}\left(G \times S^{1}, \partial G \times S^{1}\right)
$$

defined via multiplying with the $S^{1}$ factor. Moreover, all elements in $\sigma(\mathcal{V})$ are represented by surfaces which are disjoint from $K$, hence $\sigma \mid \mathcal{V}$ induces an injective map

$$
\tilde{\sigma}: \mathcal{V} \rightarrow H_{2}(X, \partial X ; \mathbb{Q}) .
$$

We pick two elements $\varepsilon_{\infty}^{\prime} \in \rho_{\infty}^{-1}(e(\mathscr{E}(\infty))), \varepsilon_{\alpha}^{\prime} \in \rho_{\alpha}^{-1}(e(\mathscr{E}(\alpha)))$. If $\operatorname{dim} \mathcal{V}>1$, then there exists a nonzero integral element $h \in \widetilde{\sigma}(\mathcal{V})$ such that

$$
\left\langle\varepsilon_{\infty}^{\prime}, h\right\rangle=\left\langle\varepsilon_{\alpha}^{\prime}, h\right\rangle .
$$

Let $H \subset X$ be a proper surface representing $h$ such that $H \cap T=\emptyset$. We claim that this $H$ is what we need. We only need to check 3 ) since the first two conditions are obvious.

From the Mayer-Vietoris sequence

$$
H^{1}(K(\xi)) \longrightarrow H^{2}(X, \partial X) \stackrel{\rho_{\xi}}{\longrightarrow} H^{2}(X(\xi), \partial X(\xi))
$$

and the fact that $h \cdot[T]=0$ we conclude that $\left\langle\varepsilon_{\xi}, h\right\rangle$ does not depend on the choice of $\varepsilon_{\xi} \in \rho_{\xi}^{-1}(e(\mathscr{E}(\xi)))$. Hence 3$)$ holds.

Assume the dimension of $\mathcal{V}$ is greater than 1 , let $H$ be a surface as in Lemma 4.4, and suppose $H \pitchfork G$. Without loss of generality, we can assume no component of $C=H \cap G$ is nullhomologous in $H_{1}(G, \partial G)$, and $H$ is efficient in $G \times S^{1}$, hence we can also assume $H \cap G$ is efficient in $G$.

Let $p \in G \backslash C$ be a point. Let $\mathcal{S}_{m}(+C)$ be the set of properly embedded oriented surfaces $S \subset G \times I$, such that $S \cap K=\emptyset, \partial_{0} S=-C \times 0, \partial_{1} S=C \times 1$, and the algebraic intersection number between $S$ and $p \times I$ is $m$. Similarly, let $\mathcal{S}_{m}(-C)$ be the set of properly embedded surfaces $S \subset G \times I$, such that $S \cap K=\emptyset, \partial_{0} S=C \times 0, \partial_{1} S=-C \times 1$, and the algebraic intersection number of $S$ with $p \times I$ is $m$. Since $[C] \cdot i_{*}([K])=0, \mathcal{S}_{m}( \pm C) \neq \emptyset$.

Suppose $S \subset M$ is a properly embedded surface which is transverse to $\partial G \times 0$. For any component $S_{0}$ of $S$, we define

$$
y\left(S_{0}\right)=\max \left\{\frac{\left|S_{0} \cap(\partial G \times 0)\right|}{2}-\chi\left(S_{0}\right), 0\right\},
$$

and let $y(S)$ be the sum of $y\left(S_{i}\right)$ with $S_{i}$ running over all components of $S$. Let $y\left(\mathcal{S}_{m}( \pm C)\right)$ be the minimal value of $y(S)$ for all $S \in \mathcal{S}_{m}( \pm C)$.

Lemma 4.5. When $m$ is sufficiently large, there exist surfaces $S_{1} \in \mathcal{S}_{m}(+C)$ and $S_{2} \in \mathcal{S}_{m}(-C)$, such that they are taut.

Proof. Let $x(\cdot)$ be the Thurston norm in $H_{2}(X, \partial X)$. There exists $N \geq 0$, such that if $k>N$, then $x([H]+(k+1)[G])=x([H]+k[G])+x(G)$. As in the proof of Gabai [2, Theorem 3.13], if $\bar{Q}$ is a Thurston norm minimizing surface in the 
homology class $[H]+k[G]$, and $\bar{Q} \cap G$ consists of essential curves in $G$, then $Q$ gives a taut decomposition of $M$, where $Q$ is obtained from $\bar{Q}$ by cutting open along $\bar{Q} \cap G$. Moreover, we can assume $\bar{Q}$ is efficient in $X$. Hence $\bar{Q} \cap T=\emptyset$ and for each boundary component $\delta$ of $G \times i,|\partial Q \cap \delta|=|[\partial Q] \cdot[\delta]|$.

Now we can apply Gabai [3, Lemma 0.6] to get a new taut surface $Q^{\prime}$ such that $\partial_{0} Q^{\prime}=-C \times 0, \partial_{1} Q^{\prime}=C \times 1$. When $m$ is sufficiently large, let $S_{1}$ be the surface obtained by doing oriented cut-and-pastes of $Q^{\prime}$ with $\left(m-Q^{\prime} \cdot(p \times I)\right)$ copies of $G$, then $S_{1} \in \mathcal{S}_{m}(+C)$ is the surface we need. Similarly, we can find the surface $S_{2} \in \mathcal{S}_{m}(-C)$.

Correction 4.6. In Ni 9], after the statement of Proposition 3.4, the author claims that there exists a circle or arc $C \subset G$ such that $[C] \cdot i_{*}[K]=0$. This claim is not true. The correct statement should be there exists an essential efficient curve $C$ in $G$ such that $[C] \cdot i_{*}[K]=0$. The proof only needs slight changes: one can make use of the above Lemma 4.5 to find taut surfaces.

The following result is $\mathrm{Ni}$ [9, Lemma 3.6], whose proof uses the assumption that $(M, \gamma)$ contains no essential product disks or product annuli and an argument from Gabai 4 .

Lemma 4.7. For any positive integers $p, q$,

$$
y\left(\mathcal{S}_{p}(+C)\right)+y\left(\mathcal{S}_{q}(-C)\right)>(p+q) y(G) .
$$

Proof of Proposition 4.3. By Lemma 4.5, when $m$ is large there exist taut surfaces $S_{1} \in \mathcal{S}_{m}(+C), S_{2} \in \mathcal{S}_{m}(-C)$. By Theorem 2.9, $S_{i}$ remains taut in $M\left(\xi_{i}\right)$ for some $\xi_{i} \in\{\infty, \alpha\}, i=1,2$. Let $\mathscr{F}_{i}$ be a taut foliation of $X\left(\xi_{i}\right)$ induced by $S_{i}$.

Let $\overline{S_{1}}, \overline{S_{2}} \subset X$ be the surfaces obtained from $S_{1}, S_{2}$ by gluing $C \times 0$ to $C \times 1$. We have

$$
\left[\overline{S_{1}}\right]=[H]+m[G], \quad\left[\overline{S_{2}}\right]=-[H]+m[G]
$$

in $H_{2}(X, \partial X)$ and $H_{2}(X(\xi), \partial X(\xi))$.

We have

$$
\chi\left(\overline{S_{i}}\right)=\chi\left(S_{i}\right)-\left|\partial_{0} S_{i}\right|=-y\left(S_{i}\right)
$$

and by Proposition 2.12

$$
\begin{aligned}
\chi\left(\overline{S_{1}}\right) & =\left\langle e\left(\mathscr{F}_{1}\right),\left[\overline{S_{1}}\right]\right\rangle \\
& =\left\langle e\left(\mathscr{E}\left(\xi_{1}\right)\right),[H]+m[G]\right\rangle, \\
\chi\left(\overline{S_{2}}\right) & =\left\langle e\left(\mathscr{F}_{2}\right),\left[\overline{S_{2}}\right]\right\rangle \\
& =\left\langle e\left(\mathscr{E}\left(\xi_{2}\right)\right),-[H]+m[G]\right\rangle .
\end{aligned}
$$

By Lemma 4.4, $\left\langle e\left(\mathscr{E}\left(\xi_{1}\right)\right),[H]\right\rangle=\left\langle e\left(\mathscr{E}\left(\xi_{2}\right)\right),[H]\right\rangle$. So

$$
\begin{aligned}
\chi\left(\overline{S_{1}}\right)+\chi\left(\overline{S_{2}}\right) & =\left\langle\mathscr{E}\left(\xi_{1}\right), m[G]\right\rangle+\left\langle\mathscr{E}\left(\xi_{2}\right), m[G]\right\rangle \\
& =2 m \chi(G),
\end{aligned}
$$

which contradicts Lemma 4.7 . 
Proof of Proposition 4.1. By Proposition 4.3, $b_{1}(G) \leq 2$, so $G$ is an annulus, a pair of pants or a genus-one surface with one boundary component. We only need to show that the last case is not possible.

Suppose $g(G)=1$ and $|\partial G|=1$. Let $C \subset G$ be a simple closed curve such that $[C] \cdot i_{*}[K]=0$, then there exists a closed taut surface $H \subset X$ such that $[H]=\left[C \times S^{1}\right]$ and $H \cap T=\emptyset$. Since $M$ does not contain any product annuli, $H$ is not a torus, hence $H$ is not taut in $X(\infty)$. By Theorem 2.9, $H$ is taut in $X(\alpha)$.

Consider the monodromy $\varphi$ of $X(\alpha)$, the surface $H \subset X(\alpha)$ forces $\varphi_{*}[C]=$ $[C]$. Since $G$ is a once-punctured torus, $\varphi(C)$ is isotopic to $C$. Thus there exists a torus $R \subset X(\alpha)$ such that $R \cap G=C=H \cap G$, which implies that $[H]=[R]+m[G]$ for some integer $m$. Since $H$ is closed, $m=0$. This contradicts the facts that $H$ is taut in $X(\alpha)$ and that $H$ is not a torus.

\section{Knots in pants $\times I$}

In this section, we study the case where $G$ is a pair of pants.

The following elementary observation is stated without proof.

Lemma 5.1. Suppose $C_{1}, C_{2} \subset G$ are two efficient curves consisting of essential arcs. If they are homologous in $H_{1}(G, \partial G)$, then they are isotopic.

Let $a, b, c$ be the three boundary components of $G, u, v, w$ be three mutually disjoint oriented arcs in $G$ such that $u$ connects $b$ to $c, v$ connects $c$ to $a, w$ connects $a$ to $b$. Then

$$
[u]+[v]+[w]=0 \in H_{1}(G, \partial G) .
$$

Lemma 5.2. None of $u, v, w$ has zero intersection number with $i_{*}[K]$.

Proof. The argument is similar to the once-punctured torus case of Proposition 4.1. Assume that $[u] \cdot i_{*}[K]=0$, then there exists a closed taut surface $H \subset X$ such that $[H]=\left[u \times S^{1}\right]$. We may assume that $H$ is efficient in $X$, hence $H$ has two boundary components and $H \cap T=\emptyset$. By Lemma 5.1, we may assume that $H \cap G=u$.

Since $M$ does not contain any product disks, $H$ is not an annulus, hence $H$ is not taut in $X(\infty)=G \times S^{1}$. By Theorem 2.9, $H$ is taut in $X(\alpha)$. Let $\varphi$ be the monodromy of $X(\alpha)$, then $H$ forces $\varphi(u)$ to be homologous hence isotopic to $u$ by Lemma 5.1. Thus there exists an annulus $A \subset X(\alpha)$ such that $A \cap G=u=H \cap G$, which implies that $[H]=[A]+m[G]$ for some integers. Since $H$ has only two boundary components, $m=0$. This contradicts the facts that $H$ is taut in $X(\alpha)$ and $H$ is not an annulus.

Lemma 5.3. The intersection number of $i_{*}[K]$ with each of $u, v, w$ is \pm 1 or \pm 2 . 


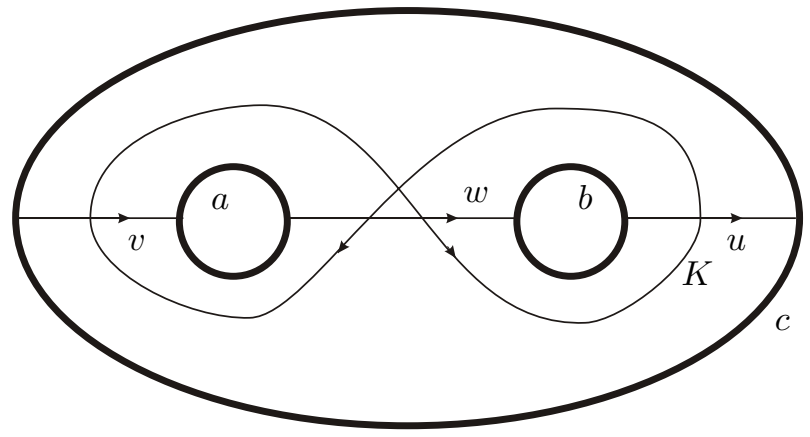

Figure 3: The homology class of $K$ in $G \times I$

Proof. Capping off $a$ with a disk, we get an annulus $G_{a}$. Now $K \subset G_{a} \times I$ and the $\alpha$-surgery on $K$ does not change the homeomorphism type of the pair $\left(G_{a} \times I,\left(\partial G_{a}\right) \times I\right)$. By the previous lemma, $K$ is nontrivial in $G_{a} \times I$. By Lemma 3.1, $K$ is the core or the $(2, \pm 1)$-cable in $G_{a} \times I$, so $i_{*}[K] \cdot[u]$ is \pm 1 or \pm 2 . The same argument applies to $v$ and $w$.

Using the previous two lemmas and (6), we may assume

$$
[u] \cdot i_{*}[K]=[v] \cdot i_{*}[K]=1, \quad[w] \cdot i_{*}[K]=-2,
$$

after reversing the orientation of $K$ and renumbering $a, b, c, u, v, w$ if necessary. We give $a, b, c$ the boundary orientation induced from $G$, then

$$
[v] \cdot[a]=[w] \cdot[b]=-[u] \cdot[b]=[u] \cdot[c]=1 .
$$

See Figure 3 for the homology class of $K$.

Let $\tau_{a}, \tau_{b}, \tau_{c}$ be the right-hand Dehn twists along (parallel copies of) $a, b, c$. The mapping class group $\mathcal{M C G}(G, \partial G)$ of $G$ is generated by $\tau_{a}, \tau_{b}, \tau_{c}$. (See, for example, Farb-Margalit [1] for preliminaries on the mapping class groups of surfaces with boundary.) Since $a, b, c$ are disjoint, $\mathcal{M C G}(G, \partial G) \cong \mathbb{Z}^{3}$.

Lemma 5.4. If $K$ is the $(2,1)$-cable in $G_{c} \times I$, then the map induced by the $\alpha$-surgery is

$$
\varphi_{\alpha}=\tau_{a}^{2} \tau_{b}^{2} \tau_{c}^{-1} .
$$

If $K$ is the $(2,-1)$-cable in $G_{c} \times I$, then

$$
\varphi_{\alpha}=\tau_{a}^{-2} \tau_{b}^{-2} \tau_{c}
$$

Proof. Capping off $a, b$ with two disks, $G$ becomes a disk $G_{a b}$. $K$ has a canonical frame $\lambda$, which is null-homologous in $\left(G_{a b} \times I\right) \backslash K$. Hence $\lambda$ is homologous to $l[a]+m[b]$ in $M$ for some integers $l, m$. By (7), (8) we conclude that $\lambda$ is homologous to $a-b$ in $M$. Hence $\lambda$ is also the canonical frame in $G_{c} \times I$, where $G_{c}$ is obtained from $G$ by capping off $c$ with a disk. 


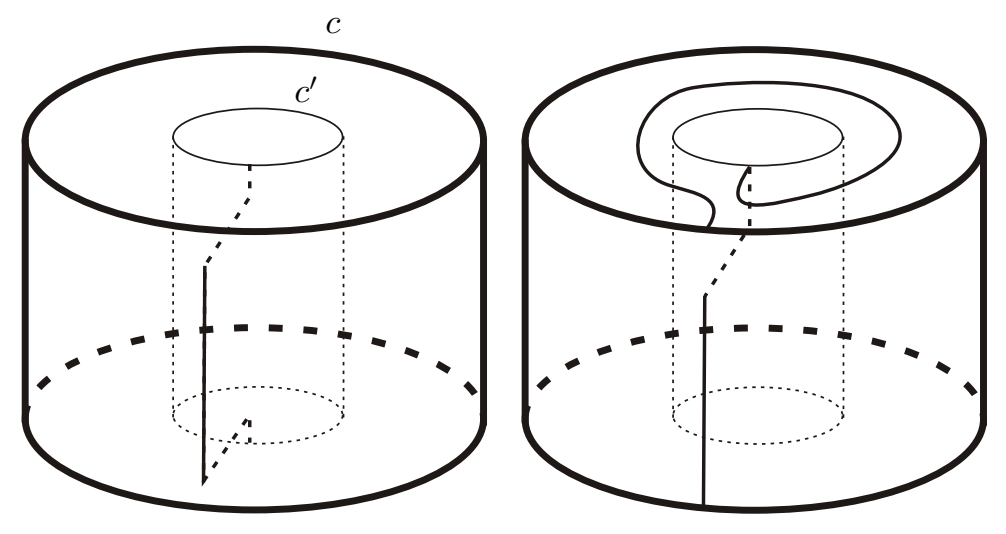

Figure 4: Local pictures of $X(\alpha)$ near $c \times S^{1}$

Suppose $\varphi_{\alpha}=\tau_{a}^{p} \tau_{b}^{q} \tau_{c}^{r}$. If $K$ is the $(2,1)$-cable in $G_{c} \times I$, then by Lemma 3.1 the slope $\alpha$ is 1 with respect to $\lambda$.

There is a natural map

$$
q_{a}: \operatorname{MCG}(G, \partial G) \rightarrow \mathcal{M C G}\left(G_{a}, \partial G_{a}\right),
$$

where $\mathcal{M C G}\left(G_{a}, \partial G_{a}\right)$ is generated by $\tau_{b}$. Since $K$ is the core in $G_{a} \times I$ and the slope $\alpha$ is $1, q_{a}\left(\varphi_{\alpha}\right)$ must be $\tau_{b}$ by Lemma 3.2. The map $q_{a}$ sends both $\tau_{b}$ and $\tau_{c}$ to $\tau_{b}$, and sends $\tau_{a}$ to 1 . So $q_{a}\left(\varphi_{\alpha}\right)=\tau_{b}^{q+r}$, thus $q+r=1$. The same argument shows that $p+r=1$.

Now consider the natural map

$$
q_{c}: \mathcal{M C G}(G, \partial G) \rightarrow \mathcal{M C G}\left(G_{c}, \partial G_{c}\right)=\left\langle\tau_{a}\right\rangle .
$$

By Lemma 3.2, $q_{c}\left(\varphi_{\alpha}\right)=\tau_{a}^{4}$. Hence $p+q=4$. So we conclude that $p=q=$ $2, r=-1$. The same argument works when $K$ is the $(2,-1)$-cable in $G_{c} \times I$.

Proposition 1.4 follows from the above lemma.

The manifold $G \times S^{1}$ has a unique product structure. Let $\omega, \omega_{\alpha} \subset c \times$ $S^{1}$ be $S^{1}$-fibres with respect to the product structures on $X(\infty)$ and $X(\alpha)$, respectively.

Lemma 5.5. If $K$ is the $(2,1)$-cable in $G_{c} \times I$, then

$$
\left[\omega_{\alpha}\right]=[\omega]+[c]
$$

Proof. The manifold $X(\infty)$ is obtained from $G \times I$ by identifying $(x, 0)$ with $(x, 1)$ for each $x \in G$. By Lemma 1.3, $X(\alpha)$ is obtained from $G \times I$ by identifying $(x, 0)$ with $\left(\varphi_{\alpha}(x), 1\right)$ for each $x \in G$. Choose parallel copies of $a, b, c$ in $G$, denoted $a^{\prime}, b^{\prime}, c^{\prime}$. Let $\varphi_{\alpha}$ be supported in the three annuli bounded by $a-a^{\prime}$, $b-b^{\prime}$ and $c-c^{\prime}$. Pick points $p \in c, p^{\prime} \in c^{\prime}$, then $p^{\prime} \times S^{1}$ is an $S^{1}$-fibre of the product structures on both $X(\infty)$ and $X(\alpha)$, while $p \times S^{1}$ is an $S^{1}$-fibre of the product structure on $X(\infty)$. 
In $X(\alpha)$, we isotope $p^{\prime} \times S^{1}$ such that it becomes a curve $\mathcal{S}$ which is the union of four segments $J, J_{\epsilon}, J_{1-\epsilon}, J^{\prime}$, where $J$ is a vertical segment in the interior of $c \times I, J_{\epsilon} \subset G \times \epsilon, J_{1-\epsilon} \subset G \times(1-\epsilon), J^{\prime}$ is a vertical segment in $c^{\prime} \times S^{1}$. See the left hand side of Figure 4 .

As on the right hand side of Figure 4, we push the previous curve $\mathcal{S}$ down in distance $\epsilon$ to get a new curve $\mathcal{S}_{-}$, then $J_{\epsilon}$ becomes an arc on $G \times 1$. Using Lemma 1.3, this new arc is $\varphi_{\alpha}\left(J_{\epsilon}\right)=\tau_{c}^{-1}\left(J_{\epsilon}\right) . \mathcal{S}_{-}$is a fibre of $X(\alpha)$, and it is homologous to $\left[p \times S^{1}\right]+[c \times 1]$. Hence our conclusion holds.

Lemma 5.6. Let $C=v-u$. Pick a point $p \in c \backslash(\partial C)$, we can then define $\mathcal{S}_{m}( \pm C)$ as in Section 4. Then there exists a connected surface $S \in S_{1}(C)$ such that $y(S)=1$. Moreover, Let $S^{\prime} \subset G \times[0,1]$ be the surface obtained from $-C \times I$ and $G \times 0$ by oriented cut-and-pastes, then $S$ is isotopic to $S^{\prime}$ in $G \times[0,1]$.

Proof. For any homology class $h \in H_{2}\left(X,(\partial G) \times S^{1}\right)$, let $x(h), x_{\infty}(h), x_{\alpha}(h)$ denote its Thurston norm in $X, X(\infty), X(\alpha)$, respectively.

Let $U=-\left[u \times S^{1}\right], V=-\left[v \times S^{1}\right] \in H_{2}\left(G \times S^{1},(\partial G) \times S^{1}\right)$. Since $(V-$ $U) \cdot[K]=0, V-U$ also represents an element in $H_{2}\left(X,(\partial G) \times S^{1}\right)$. Note that the Thurston norm of $h \in H_{2}\left(G \times S^{1},(\partial G) \times S^{1}\right)$ is the absolute value of its algebraic intersection number with the $S^{1}$-fibre. Consider $V-U+m[G]$ for $m \geq 0$, using Lemma [5.5, we can compute

$$
\begin{gathered}
x_{\infty}(V-U+m[G])=m, \\
x_{\alpha}(V-U+m[G])=(V-U+m[G]) \cdot([\omega]+[c])=|m-2| .
\end{gathered}
$$

Since $x_{\infty}(V-U+[G])=x_{\alpha}(V-U+[G])=1$, Theorem 2.9 implies that $x(V-U+[G])=1$. Let $\bar{S} \subset X$ be a taut surface in this homology class such that $\bar{S}$ is efficient in $X$. Then $\bar{S}$ is disjoint from $T$. Isotope $\bar{S}$ so that it is transverse to $G$ and its intersection with $G$ contains no trivial loops. Now $\bar{S} \cap G$ is homologous to $C$. Moreover, $\bar{S} \cap G$ can be made efficient in $G$. So $\bar{S} \cap G$ is isotopic to $C$ by Lemma [5.1. Without loss of generality, we can assume

$$
\bar{S} \cap G=C \quad \text { and } \quad \bar{S} \cap\left((\partial C) \times S^{1}\right) \subset G .
$$

Cutting $\bar{S}$ open along $C$, we get a surface $S \in \mathcal{S}_{1}(+C)$ such that $y(S)=1$. After an isotopy of $S$, we can assume the two surfaces $S, C \times[0,1] \subset G \times[0,1]$ are transverse. Since $S \cap((\partial C) \times(0,1))=\emptyset, S \cap(C \times(0,1))$ consists of closed curves which bounds disks in $C \times(0,1)$. Since $S$ is incompressible and $G \times[0,1]$ is irreducible, we can isotope $S$ such that $S \cap(C \times(0,1))=\emptyset$, hence $S \cap(C \times[0,1])=$ $C \times\{0,1\}$. Now we glue $S$ and $C \times[0,1]$ together along $C \times\{0,1\}$ and perturb the resulting surface slightly, then we get a connected surface $G^{\prime}$ with $x\left(G^{\prime}\right)=1$ and $\partial G^{\prime}$ is parallel to $(\partial G) \times 0$ in $(\partial G) \times[0,1]$. Hence $G^{\prime}$ is parallel to $G \times 0$ in $G \times[0,1]$. It follows that $S$ is isotopic to $S^{\prime}$ in $G \times[0,1]$.

Lemma 5.7. Let $S$ be the surface obtained in Lemma 5.6. Let

$$
G \times I \stackrel{S}{\rightsquigarrow}\left(M_{1}(\infty), \gamma_{1}\right)
$$


be the sutured manifold decomposition associated with $S$, then $\left(M_{1}(\infty), \gamma_{1}\right)$ is a product manifold, and there is an ambient isotopy of $M_{1}(\infty)$ which takes $K$ to a curve in $R_{+}\left(\gamma_{1}\right)$ such that the frame of $K$ specified by $R_{+}\left(\gamma_{1}\right)$ is $\alpha$.

Proof. By Lemma 5.6, $S$ is obtained from $-C \times I$ and $G \times 0$ by oriented cutand-pastes. So $\left(M_{1}(\infty), \gamma_{1}\right)$ is a product sutured manifold and $R_{+}\left(\gamma_{1}\right)$ is an annulus.

Let $\left(M_{1}(\alpha), \gamma_{1}\right)$ be the sutured manifold obtained from $M(\alpha)$ by decomposing along $S$. Then $M_{1}(\alpha)$ can also be obtained from $M_{1}(\infty)$ by $\alpha$-surgery on $K$.

We claim that $M_{1}(\alpha)$ is not taut. In fact, let $S^{\prime \prime}$ be the surface obtained from $S$ and $G \times 0$ by oriented cut-and-pastes. Let $\overline{S^{\prime \prime}} \subset X$ be the surface obtained from $S^{\prime \prime}$ by gluing $\partial_{0} S^{\prime \prime}$ to $\partial_{1} S^{\prime \prime}$. Then $x\left(\overline{S^{\prime \prime}}\right)=2$ and $\overline{S^{\prime \prime}}$ represents $V-U+2[G]$. We already computed

$$
x_{\infty}(V-U+2[G])=2>x_{\alpha}(V-U+2[G])=0,
$$

so $\overline{S^{\prime \prime}}$ is not taut in $X(\alpha)$. Let $M^{\prime \prime}(\alpha)$ be the non-taut sutured manifold obtained by decomposing $X(\alpha)$ along $\overline{S^{\prime \prime}}$.

Since $S^{\prime \prime}$ is obtained from $S$ and $G \times 0$ by oriented cut-and-pastes, and $S \cap(G \times 0)=-C \times 0$ consists of two arcs, there exist two product disks in $M^{\prime \prime}(\alpha)$ such that the result of decomposing $M^{\prime \prime}(\alpha)$ along these two disks is $\left(M_{1}(\alpha), \gamma_{1}\right)$. See the proof of Gabai [2, Theorem 3.13] for an explanation of this fact. So $\left(M_{1}(\alpha), \gamma_{1}\right)$ is not taut by Gabai [3, Lemma 0.4].

Now Theorem 1.5 implies our conclusion.

Proof of Theorem 1.1. By the results in Sections 3 and 4 we only need to consider the case $F=G$ is a pair of pants. By Lemma 5.7 $K$ lies on $R_{+}\left(\gamma_{1}\right)$, and the frame specified by $R_{+}\left(\gamma_{1}\right)$ is $\alpha$.

Since $R_{+}\left(\gamma_{1}\right)$ is an annulus, the only essential curve on it is its core. As in Figure 5, $R_{+}\left(\gamma_{1}\right)$ can be constructed in the following way. Cut $G \times\{0,1\}$ open along $(v-u) \times\{0,1\}$, we get two octagons $P_{0}, P_{1}$. There are two edges of $P_{0}$ which are copies of $v \times 0$ with different orientations. We call these two edges $v \times 0,-v \times 0$. Similarly, there are edges $\pm u \times 0, \pm v \times 1, \pm u \times 1$. Now we glue two product disks to $P_{0}, P_{1}$, such that one product disk connects $v \times 0$ to $-v \times 1$ and the other connects $-u \times 0$ to $u \times 1$. The annulus we get is isotopic to $R_{+}\left(\gamma_{1}\right)$. The core of this annulus is clearly a one-crossing knot in $G \times I$. The result about the frame also follows since the vertical projection $p: R_{+}\left(\gamma_{1}\right) \rightarrow G$ is an immersion.

\section{References}

[1] B. Farb, D. Margalit, A primer on mapping class groups, to appear in Princeton Mathematical Series, Princeton University Press, available at http://www.math.utah.edu/ ${ }^{\sim}$ margalit/primer/. 


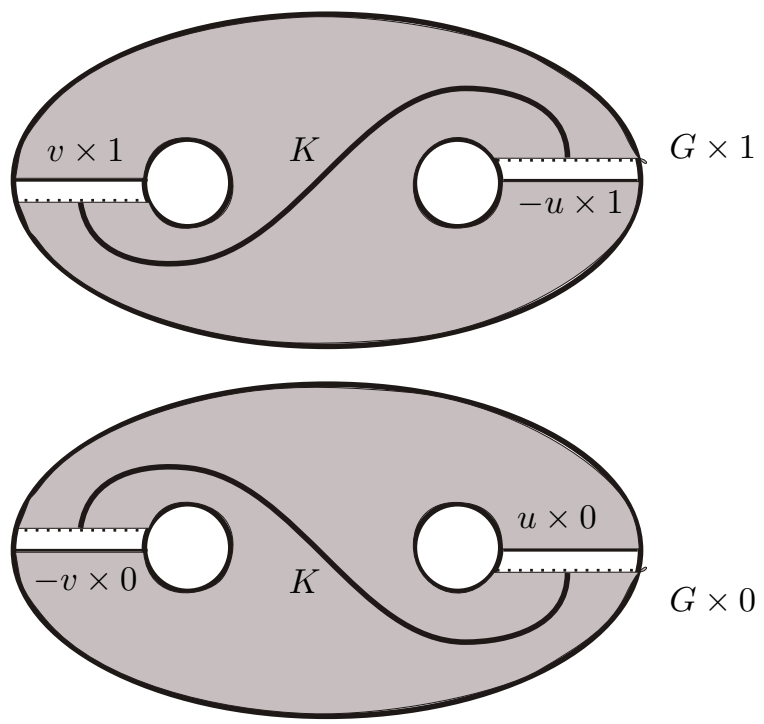

Figure 5: The surface $R_{+}\left(\gamma_{1}\right)$ containing the knot $K$

[2] D. Gabai, Foliations and the topology of 3-manifolds, J. Differential Geom. 18 (1983), no. 3, 445-503.

[3] D. Gabai, Foliations and the topology of 3-manifolds II, J. Differential Geom. 26 (1987), no. 3, 461-478.

[4] D. Gabai, Foliations and the topology of 3-manifolds III, J. Differential Geom. 26 (1987), no. 3, 479-536.

[5] D. Gabai, Surgery on knots in solid tori, Topology 28 (1989), no. 1, 1-6.

[6] D. Gabai, 1-bridge braids in solid tori, Topology Appl. 37 (1990), no. 3, 221-235.

[7] P. Ghiggini, Knot Floer homology detects genus-one fibred knots, Amer. J. Math. 130 (2008), no. 5, 1151-1169.

[8] C. Gordon, J. Luecke, Knots are determined by their complements, J. Amer. Math. Soc. 2 (1989), no. 2, 371-415.

[9] Y. Ni, Dehn surgeries that yield fibred 3-manifolds, Math. Ann. 344 (2009), no. $4,863-876$.

[10] D. Rolfsen, Knots and links, Mathematics Lecture Series, No. 7, Publish or Perish, Inc., Berkeley, Calif., 1976.

[11] M. Scharlemann, A. Thompson, Heegaard splittings of (surface) $\times I$ are standard, Math. Ann. 295 (1993), no. 3, 549-564. 
[12] M. Scharlemann, A. Thompson, Surgery on a knot in (Surface $\times I$ ), Algebr. Geom. Topol. 9 (2009), 1825-1835.

[13] W. Thurston, A norm for the homology of 3-manifolds, Mem. Amer. Math. Soc. 59 (1986), no. 339, i-vi and 99-130. 